\title{
Electrical Activation Enhances Pre-Implantation Embryo Development Following Sperm Injection into In Vitro Matured Pig Oocytes
}

\author{
Jae-Gyu YOO ${ }^{1)}$, Chang-Gi HUR ${ }^{2)}$, Mi-Rung PARK ${ }^{3)}$, Jong-Yi PARK ${ }^{3)}$, Kyu-Chan HWANG ${ }^{3)}$, Jae-Hwan KIM ${ }^{4}$, \\ Jin-Hoi $\mathrm{KIM}^{3)}$ and Seong-Keun $\mathrm{CHO}^{5) *}$ \\ ${ }^{1)}$ Division of Animal Science, National Institute of Animal Science, Rural Development Administration, Suwon, Gyeonggi 441-706, \\ Republic of Korea \\ 2) CHO-A Biotechnology Research Institute, CHO-A Pharmaceutical Co. Ltd., Seoul 150-992, Republic of Korea \\ 3) Department of Animal Biotechnology, KonKuk University, Seoul 143-701, Republic of Korea \\ 4) Department of Biomedical Science, College of Life Science, CHA University, Pochon, Gyeonggi 487-010, Republic of Korea \\ ${ }^{5)}$ Department of Animal Science, Pusan National University, Miryang, Gyeongnam 627-706, Republic of Korea
}

(Received 23 February 2011/Accepted 13 November 2011/Published online in J-STAGE 25 November 2011)

ABSTRACT. The objective of this study was to evaluate the effect of electrical stimulation (EST) on pronuclear formation, chromosomal constitution, and developmental capability among in vitro matured pig oocytes following intracytoplasmic sperm injection (ICSI). After ICSI, the oocytes were randomly distributed and cultured into 3 groups: the EST activated ICSI group, non-activation ICSI group, and in vitro fertilization (IVF) group. The proportion of oocytes in which 2 pronuclei were formed in ICSI groups was significantly higher in the former groups than in the IVF group ( 96.2 and 93.5 vs. $64.5 \%$, respectively, $P<0.05$ ). The cleavage rate was significantly higher in EST activated ICSI group (78.6\%) than in the IVF and non-activated ICSI groups (51.8 and $46.0 \%$, respectively, $P<0.05$ ), as was the proportion of oocytes that developed to the blastocyst stage at day 7 (18.9 vs. 11.6 and $9.1 \%$, respectively, $P<0.05$ ). Diploid blastocysts were observed in $52.4,63.0$, and $65.2 \%$ of oocytes in the IVF, activated, and non-activated ICSI groups, respectively. Eight out of 23 gilts (34.8\%) were confirmed to be pregnant in activated ICSI groups, but none of these pregnancies were carried to term. These results show that oocyte activation after ICSI is effective in elevating the cleavage rate and blastocyst development, while ensuring normal chromosome composition. Further research is needed to determine the pregnancy maintenance requirements for ICSI-embryos in pigs.

KEY WORDS: activation, ICSI, IVM, oocyte, swine.

doi: 10.1292/jvms.11-0073; J. Vet. Med. Sci. 74(4): 429-434, 2012

Intracytoplasmic sperm injection (ICSI) is a valuable assisted reproduction technology for studying fertilization, treating human infertility, and producing transgenic animals. In humans, ICSI has become a widely applied means of overcoming infertility. In addition to its clinical usefulness, ICSI can be a valuable research tool for studying the fundamental aspects of interaction between 2 gametes during fertilization. Since the first report of the use of ICSI in mammals [38], several successful attempts to produce live offspring with ICSI using both in vivo matured [27, 37] and in vitro matured [2] oocytes have been reported. In pigs, successful production of offspring after ICSI has been reported mostly with in vivo matured oocytes $[12,21]$. The use of ICSI-fertilized porcine in vitro matured oocytes resulted in the death of a piglet immediately after birth [17]. Recently, Nakai et al. [24] reported 3 healthy piglets produced by ICSI for the first time using in vitro matured porcine oocytes. In several species such as rabbits [19], hamsters [5], and mice [15, 26], injection

*Correspondence to: Cho, S.-K., Department of Animal Science, Pusan National University, Miryang, Gyeongnam 627706, Republic of Korea.

e-mail: skcho@pusan.ac.kr

(C)2012 The Japanese Society of Veterinary Science of the sperm itself is apparently sufficient to activate the oocyte, as the sperm nucleus can undergo decondensation and form a pronucleus (PN).

Compared with other species, the ICSI procedure has not been well established in pigs, partially because of activation of in vitro matured oocytes after ICSI, which lowers the fertilization rate and results in abnormal development of ICSI-fertilized porcine and bovine oocytes [18]. Thus, it appears that sperm preparation and post-ICSI oocyte activation affect the fertilization and development of ICSIderived embryos [3, 11, 35]. Spermatozoa derived from several mammalian species are also unable to activate oocytes, mainly due to lack of oocyte activation factors. Recent studies have shown that species differences exist in oocyte activation after ICSI. An additional activation procedure is required to allow oocyte fertilization following spermatozoa injection in cattle [35] and pigs [18], whereas injection alone is sufficient to activate oocytes in mice and humans [37]. Although mechanical stimulation by the injection pipette occasionally results in activation, further stimulation is needed for porcine oocytes. Activation of mammalian oocytes in several species has been attempted using various chemicals, as well as physical and enzymatic stimuli [7]. Similar attempts have been made to improve ICSI results in pigs and cattle [11, 35], and stimuli such as ethanol $[13,22,23]$, calcium ionophore $[4,11,40]$ and 
EST $[1,34,40]$ have been found to activate oocytes. In pigs, the blastocyst formation rate of ICSI-fertilized oocytes is significantly enhanced by electrical activation [17, 18], and EST is a potent activator of porcine oocytes [31, 40, 41]. Since an electrical stimulus can increase the oocyte $\mathrm{Ca}^{2+}$ concentration, it has often been used to activate porcine oocytes after nuclear transfer [20, 25, 32] and ICSI [17], and in parthenogenetic oocytes [41]. However, little is known about the effect of EST on embryo development in pigs.

In this study, we investigated the effect of electrical stimulation (EST) after sperm injection into in vitro matured pig oocytes on early and later embryo development. The observations indicate that EST activation of porcine oocytes after ICSI plays a crucial role in the formation of male and female pronuclei and the subsequent cleavage and development of in vitro matured oocytes. In this study, we present evidence that the efficiency of porcine ICSI can be improved by oocyte activation by EST: we describe PN formation, cleavage rate, and the chromosomal constitution of embryos and their development in vitro.

\section{MATERIALS AND METHODS}

In vitro maturation of oocytes: Prepubertal porcine ovaries were collected from a local slaughterhouse and transported to the laboratory at $30^{\circ} \mathrm{C}$ in Dulbecco's phosphatebuffered saline (D-PBS) supplemented with $5.54 \mathrm{mM}$ D-glucose, $0.33 \mathrm{mM}$ sodium pyruvate, $75 \mu \mathrm{g} / \mathrm{m} l$ potassium penicillin $\mathrm{G}$, and $50 \mu \mathrm{g} / \mathrm{m} l$ streptomycin sulphate (mDPBS). Cumulus-oocyte complexes (COCs) were aspirated with an 18-gauge needle into a disposable $10-\mathrm{m} l$ syringe and follicles 3-6 $\mathrm{mm}$ in diameter were collected. The COCs were washed 3 times with TL-HEPES medium [30]. Collections of 10-20 COCs were matured in $100 \mu \mathrm{l}$ of BSA-free NCSU 23 medium [29] supplemented with $10 \%$ porcine follicular fluid, $0.6 \mathrm{mM}$ cysteine, $0.5 \mu \mathrm{g} / \mathrm{m} l \mathrm{FSH}, 0.5 \mu \mathrm{g} / \mathrm{m} l \mathrm{LH}, 1 \mu \mathrm{g} /$ $\mathrm{m} l \mathrm{E}_{2}$, and $10 n \mathrm{~g} / \mathrm{m} l$ EGF. After the first $20-22 \mathrm{hr}$ of incubation, oocytes were cultured for a further 20-22 hr in the same maturation medium without hormone supplementation. After culture, expanded cumulus cells were removed in NCSU 23 medium containing $0.1 \%$ hyaluronidase by vortexing for $3 \mathrm{~min}$.

Preparation of spermatozoa: Epididymal spermatozoa were obtained by castration of 3 boars of proven fertility. The spermatozoa were removed from the distal segment of the caudae epididymides by retrograde flushing from the ductus deferens. Spermatozoa were washed twice with TLHEPES and then suspended in $10 \mathrm{~m} l$ of TL-HEPES. To induce capacitation, the sperm were resuspended in $10 \mathrm{~m} l$ of mTBM containing $2.5 \mathrm{mM}$ caffeine in a $15-\mathrm{m} l$ conical tube and kept at $39^{\circ} \mathrm{C}$ for $30-60 \mathrm{~min}$ [12].

In vitro fertilization: Twenty matured oocytes were cultured with spermatozoa at a final concentration of $5 \times 10^{5} /$ $\mathrm{m} l$ in $50 \mu l$ droplet of mTBM supplemented with $2.5 \mathrm{mM}$ caffeine sodium benzoate and $4 \mathrm{mg} / \mathrm{ml}$ BSA for $6 \mathrm{hr}$ at $39^{\circ} \mathrm{C}$ in an atmosphere of $5 \% \mathrm{CO}_{2}$ in air. The eggs were then transferred to $50 \mu l$ droplet of NCSU23 medium supplemented with $4 \mathrm{mg} / \mathrm{m} l \mathrm{BSA}$, and cultured for 8 days at $39^{\circ} \mathrm{C}$ in an atmosphere of $5 \% \mathrm{CO}_{2}$ in air.

Spermatozoa injection into oocytes: Spermatozoa were centrifuged at $400 \mathrm{~g}$ for $5 \mathrm{~min}$ and resuspended with TLHEPES: $10 \%$ polyvinylpyrrolidone solution (1:2). Oocytes with visible polar body and excellent morphology were used for this experiment. Oocytes were centrifuged for 6 $\min$ at $12,000 \mathrm{~g}$ in $500 \mu \mathrm{l}$ TL-HEPES medium in $1.2 \mathrm{ml}$ Eppendorf centrifuge tube. The injection of spermatozoon into the oocyte cytoplasm was performed using the method of Kim et al. [8]. Briefly, the injection needle used was 6-7 $\mu \mathrm{m}$ inner and $8-9 \mu \mathrm{m}$ outer diameters. The polar body was at 6 or 12 o'clock and the point of injection at 3 o'clock. An oocyte was penetrated by the injecting micropipette, small amount of cytoplasm was drawn into the micropipette, and then the cytoplasm together with the sperm cell and small amount of medium was expelled into the oocyte. After ooplasm injection, the injecting micropipette was withdrawn immediately, and the oocytes were released from the holding pipette to reduce the intracytoplasmic pressure exerted to the oocyte.

Activation and further development of the oocytes: The procedure for EST of porcine oocytes was conducted as described by Kim et al. [8]. EST to induce activation was performed with a BTX Electro Cell Manipulator (Biotechnologies and Experimental Research, Inc., San Diego, CA, U.S.A.) to a chamber with 2 parallel platinum wire electrodes (200 $\mu \mathrm{m}$ o.d.) spaced $1 \mathrm{~mm}$ apart overlaid. After 30 min sperm injection, oocytes were stimulated by a 10 -sec pulse at $0.48 \mathrm{KV} / \mathrm{cm} \mathrm{AC}$ followed by a $30 \mu \mathrm{sec}$ pulse at $1.26 \mathrm{KV} / \mathrm{cm} \mathrm{DC}$ at room temperature $\left(25^{\circ} \mathrm{C}\right)$. After stimulation, all oocytes were washed with NCSU23 medium and transferred to a $50 \mu \mathrm{l}$ NCSU23 medium supplemented with $4 \mathrm{mg} / \mathrm{ml} \mathrm{BSA}$, and cultured for 8 days at $39^{\circ} \mathrm{C}$ in an atmosphere of $5 \% \mathrm{CO}_{2}$ in air.

Assessment of pronuclear formation and cell number of blastocysts: Pronuclear formation was classified $18 \mathrm{hr}$ after start of IVF, sperm injection, and EST, respectively. Embryos were mounted on slide and fixed in $25 \%$ (v:v) acetic alcohol for 48 to $72 \mathrm{hr}$ at room temperature, then stained with $1 \%(\mathrm{w}: \mathrm{v})$ orcein in $45 \%(\mathrm{v}: \mathrm{v})$ acetic acid. The pronuclear stages of eggs were examined under a phase-contrast microscope at a magnification of $\times 400$. After $168 \mathrm{hr}$ of in vitro embryo culture, blastocysts were fixed at room temperature for $1 \mathrm{hr}$ with $4 \%$ paraformaldehyde and stained with $0.05 \mathrm{mM}$ bisbenzimide (Hoechst 33342). The cell numbers of blastocysts were examined at $\times 200$ and $\times 400$ magnification by epifluorescence microscope (Olympus, Tokyo, Japan).

Chromosomal analysis: At $144 \mathrm{hr}$ after injection, blastocysts were cultured in culture medium in the presence of colcemid $(0.05 \mu \mathrm{g} / \mathrm{m} l)$ for $5 \mathrm{hr}$. The blastocysts were then placed in a hypotonic solution of sodium citrate $(1 \%)$ for $5 \mathrm{~min}$, and fixed individually on microscope slides by the air-drying method [36]. Blastocysts were stained with $10 \%$ Giemsa at $\mathrm{pH}$ 6.8. The chromosome constituent of each spread was determined at $\times 1,000$ magnifications under oil.

Transfer of zygotes and embryos produced by ICSI: Induction of estrus was accomplished by i.m. injection of 
Table 1. Pronuclear formation of porcine oocytes after IVF and ICSI with and without activation

\begin{tabular}{lccccc}
\hline \multirow{2}{*}{ Treatment } & \multirow{2}{*}{ No. of oocytes } & \multicolumn{4}{c}{ Pronuclear formation (\%)* } \\
\cline { 3 - 6 } & & 1PN & 2PN & $1 \mathrm{PN}+\mathrm{sPN}$ & Other \\
\hline Control (IVM-F-C) & 62 & $12(19.4)$ & $\left.40(64.5)^{\mathrm{b}}\right)$ & $4(6.5)$ & $6(9.7)$ \\
ICSI (activation**) & 52 & 0 & $\left.50(96.2)^{\mathrm{a}}\right)$ & 0 & $2(3.8)$ \\
ICSI (non-activation) & 46 & 0 & $43(93.5)^{\mathrm{a})}$ & $3(6.5)$ & 0 \\
\hline
\end{tabular}

a), b) Within column, percentages with different superscripts differ significantly, $P<0.05$. *2PNC, male and female pronuclear chromosomal structure (PNC); $1 \mathrm{PN}+\mathrm{sPN}$, condensed or swelling male chromatin; other, plate and unidentified multiple pronuclei. **Activation; $30 \mathrm{~min}$ after ICSI, 85 volts, $30 \mu \mathrm{sec}, 1$ pulse.

Table 2. In vitro development of porcine zygotes cultured for 7 days after IVF and ICSI

\begin{tabular}{lccc}
\hline Treatment & $\begin{array}{c}\text { No. of oocytes } \\
\text { used }\end{array}$ & $\begin{array}{c}\text { No. (\%) of embryos } \\
\text { cleaved }\end{array}$ & $\begin{array}{c}\text { No. (\%) of embryos } \\
\text { developed to blastocysts }\end{array}$ \\
\hline Control (IVM-F-C) & 731 & $379(51.8)^{\mathrm{b}}$ & $85(11.6)^{\mathrm{b})}$ \\
ICSI (activation**) & 355 & $279(78.6)^{\mathrm{a})}$ & $67(18.9)^{\mathrm{a})}$ \\
ICSI (non-activation) & 496 & $228(46.0)^{\mathrm{b})}$ & $45(9.1)^{\mathrm{b})}$ \\
\hline
\end{tabular}

*Percentages with different superscripts within a column differ significantly, $P<0.05$. **Activation; $30 \mathrm{~min}$ after ICSI, 85 volts, $30 \mathrm{msec}, 1$ pulse.

Table 3. Chromosomal analysis of porcine embryos produced by IVF or ICSI

\begin{tabular}{lcccr}
\hline \multirow{2}{*}{ Treatment } & No. of blastocysts & \multicolumn{3}{c}{ No. (\%) of Ploidy } \\
\cline { 3 - 5 } & identified & Haploid & Diploid & Other* \\
\hline Control (IVM-F-C) & 21 & 0 & $11(52.4)$ & $10(47.6)$ \\
ICSI (activation**) & 27 & 0 & $17(63.0)$ & $10(37.0)$ \\
ICSI (non-activation) & 23 & 0 & $15(65.2)$ & $8(34.8)$ \\
\hline
\end{tabular}

*Other includes embryos with unidentified and mixed multiple chromosomes. **Activation; $30 \mathrm{~min}$ after ICSI, 85 volts, $30 \mathrm{msec}, 1$ pulse.

1,000 IU of equine chorionic gonadotropin (eCG; Intergonan, Intervet, Boxmeer, Netherlands) followed by 500 IU of human chorionic gonadotropin (hCG; Ovogest, Intervet) $72 \mathrm{hr}$ later. Sperm-injected eggs were transferred to the oviducts. Fifty hours after the hCG injection as described by Wallenhorst and Holts [39]. Thirty eight recipients received 3,166 embryos of 1 to 4-cell-stage and pregnancy was determined on 25 and 50 days after embryo transfer by ultrasonography. In detail, 1,252 embryos of non-activated ICSI group were transferred into 15 surrogated mothers and 1,914 embryos of activated ICSI group were transferred into 23 surrogated mothers.

Statistical analysis: All percentage data were transformed to the arc-sine of their square roots. The one-way ANOVA test was used for the comparison of group mean difference. Unless otherwise stated, statistical significance was determined at $P<0.05$.

\section{RESULTS}

Pronuclear formation among in vitro fertilized and ICSI-fertilized oocytes: In Table 1, we compare pronuclear formation among 3 different groups (in vitro fertilization control, ICSI followed by activation, and ICSI without activation). The proportion of oocytes with 2 pronuclear formations was significantly higher in the ICSI groups, irrespec- tive of EST, than in the IVF group (Table 1, 96.2 and 93.5 vs. $64.5 \%$, respectively, $P<0.05)$. Some of the PN found among IVF oocytes possessed only a female PN (19.4\%).

Development of in vitro fertilized and ICSI-fertilized oocytes: Following injection of the spermatozoa, the eggs were allocated to the EST activation or non-activation groups, as described in Materials and Methods, and their cleavage and developmental rates were compared with those in the IVF group ( $\mathrm{n}=7$, Table 2). The EST activation group had a significantly higher cleavage rate (78.6\%) than the IVF and non-activation ICSI groups (51.8 and 46.0, respectively, $P<0.05)$. Development to the blastocyst stage at 7 days was also significantly higher in the activation group than in the IVF and non-activation groups (18.9 vs. 11.6 and $9.1 \%$, respectively, $P<0.05$ ).

Chromosomal analysis of embryos: Ploidy analysis of blastocysts was performed to examine the incidence of diploidy in each of the treatment groups. Among the IVF, activation, and non-activation groups, 52.4, 63.0, and 65.2\% of embryos, respectively, were diploid (Table 3).

Cell number of IVF and ICSI oocytes: The average number of blastocyst nuclei at day 8 was $46.7 \pm 2.9,44.7 \pm 4.2$, and $41.9 \pm 4.6$ in the IVF, activation, and non-activation groups, respectively (Table 4).

Transfers of zygotes and embryos produced by ICSI: Thirty-eight gilts received 40-185 ICSI embryos, and preg- 
nancy was determined by ultrasonography at days 25 and 50 of gestation. Eight out of twenty-three gilts (34.8\%) were confirmed to be pregnant in activated ICSI groups and one out of fifteen gilts $(6.7 \%)$ was pregnant in non-activated ICSI group. Butnone of these pregnancies were carried to term (Fig. 1).

\section{DISCUSSION}

In the present study, an EST procedure was added after ICSI in pigs. EST is a well known and potent activator of porcine oocytes in somatic cell nuclear transfer and ICSI $[6,14,33,40]$. After electrical stimulation, cytoplasmic centrosomal material is activated and organizes a network with microtubules that move pronuclei to the center of the oocytes [9]. We found that 2 pronuclei were formed in similar proportions in ICSI-fertilized oocytes, irrespective of whether EST was applied or not. However, EST significantly increased the rates of oocyte cleavage and blastocyst development compared with oocytes that were not activated by EST.

Induction of oocyte activation by injection of intact or isolated spermatozoa suggests that an oocyte activation factor may be present in the plasma membrane of the porcine sperm head [9]. In the pig, as in mice, oocyte-activating factors appear to be assembled during spermatogenesis. Recently, Parrington et al. [28] reported that the sperm-borne oocyte-activating factor is a 33-KDa protein, oscillin, residing in the equatorial segment of the acrosomal region. It has been suggested that an additional activation procedure is not required for normal ICSI fertilization. In mice, isolated spermatazoa heads, which lose all of their components except for the nucleus and the perinuclear materials, can activate oocytes as efficiently as intact spermatozoa when injected [15]. Kim et al. [9] demonstrated that both a spermatozoon and an isolated sperm head could activate oocytes without additional activation when injected into the cytoplasm in pigs. However, the present study demonstrated that sperm injection procedure itself without oocyte activation treatment is not sufficient to activate porcine oocytes. In this study, shame injection (three replicates) also has been conducted (data not shown in table). Forty out of eighty-five $(47.1 \%)$ oocytes were cleaved and 1 out of 85 oocytes $(2.5 \%)$ was developed to blastocyst stage. It seems that embryo development of ICSI without activation has been affected partially by physical injection procedure. EST followed by ICSI showed a significantly higher cleavage rate than the ICSI group that was not subjected to EST. We also observed that pronuclear formation was higher in post-ICSI oocytes compared with those fertilized by IVF. However, in our study, similar observations were reported by Lee et al. [18] that the cleavage failure rate was higher in ICSI alone group compared to ICSI + EST activation or EST activation + ICSI at $168 \mathrm{hr}$ after ICSI. When ICSI was combined with EST, a significantly higher blastocyst development rate was seen in activated COCs compared with ICSI alone group. EST after injection of a spermatozoon enhanced the incidence of fertilization of in vitro matured
Table 4. Number of blastomeres of in vitro-developed porcine blastocysts derived from IVF or ICSI

\begin{tabular}{lccc}
\hline \multirow{2}{*}{ Treatment } & \multirow{2}{*}{$\begin{array}{c}\text { No. of } \\
\text { blastocysts* }\end{array}$} & \multicolumn{2}{c}{ No. of blastomeres } \\
\cline { 2 - 4 } & 21 & Mean \pm SE & Ranges \\
\hline Control (IVM-F-C) & 10 & $46.7 \pm 2.9$ & $22-68$ \\
ICSI (activation) & 10 & $41.7 \pm 4.2$ & $20-64$ \\
ICSI (non-activation) & 10.6 & $27-52$ \\
\hline
\end{tabular}

*Examined at $168 \mathrm{hr}$ post-injection or insemination.

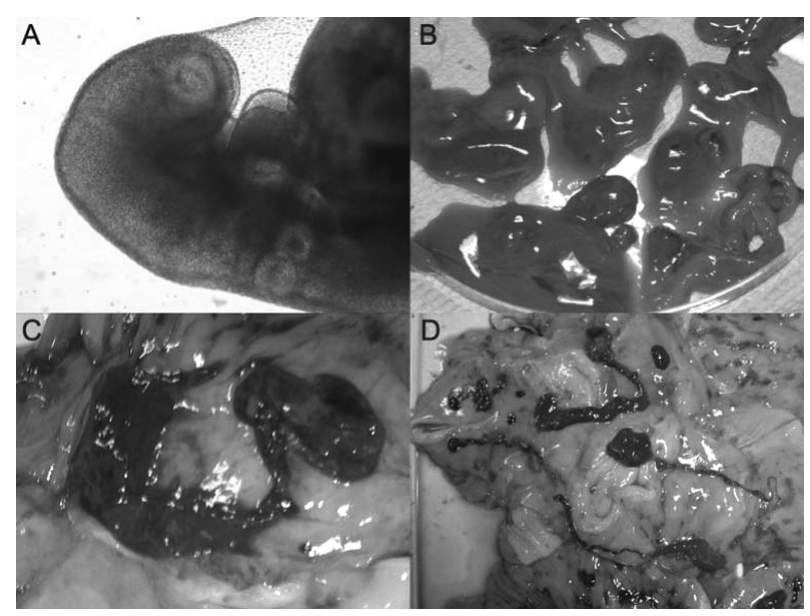

Fig. 1. Representative photographs of live and mummified porcine fetuses. A) Live fetus at 18 days. B), C), and D) Mummified fetuses at 54, 96, and 111 days of gestation.

porcine oocytes, suggesting that the timing of sperm injection and oocyte activation are important for the normal development of pronuclei and for syngamy $[10,18]$. Thus, an additional electrical activation procedure after ICSI should enhance fertilization and subsequent embryonic development in porcine oocytes.

Chromosomal analysis of blastocysts showed a similar incidence of diploidy in the IVF control and ICSI groups, irrespective of whether EST was used or not. The proportion of oocytes with multiple chromosomal abnormalities was also similar among all 3 groups. It is possible that the occurrence of chromosomal abnormalities might be due to inhibition of extrusion of the second polar body as some nuclei presumably re-enter the S-phase of the cell cycle without having passed through metaphase.

The birth of live piglets following ICSI has been reported by 2 groups [12, 15, 16, 21]. These groups have succeeded in producing piglets from laparotomized or aspirated oocytes in vivo. In this study, activated ICSI embryos transferred gilts had higher pregnancy rate than non-activated ICSI embryos transferred gilts at days 25 and 50 of gestation. It suggested that activated ICSI group had higher in vivo embryo development than non-activated ICSI group in early implantation period like in vitro embryo development comparison. However, none of the pregnancies proceeded to term, suggesting that the viability of blastocysts 
produced by ICSI is limited.

This study showed that the activation of oocytes with EST after ICSI is effective in increasing the cleavage rate and subsequent blastocyst development, while maintaining a normal chromosome composition in vitro. Further studies regarding factors involving the development of embryos after ICSI and after implantation are needed to determine how to maintain pregnancies induced by ICSI-fertilized in vitro matured porcine oocytes.

ACKNOWLEDGMENTS. This study was supported, in part, by the Agenda Program (No. PJ007577) and BioGreen 21 Program grant (Code \#20070401034017) from Rural Development Administration, Republic of Korea.

\section{REFERENCES}

1. Campbell, K. H., Ritchie, W. A. and Wilmut, I. 1993. Nuclearcytoplasmic interactions during the first cell cycle of nuclear transfer reconstructed bovine embryos: implications for deoxyribonucleic acid replication and development. Biol. Reprod. 49: 933-942. [Medline] [CrossRef]

2. Catt, S. L., Catt, J. W., Gomez, M. C., Maxwell, W. M. and Evans, G. 1996. Birth of a male lamb derived from an in vitro matured oocyte fertilised by intracytoplasmic injection of a single presumptive male sperm. Vet. Rec. 139: 494-495. [Medline] [CrossRef]

3. Devito, L. G., Fernandes, C. B., Blanco, I. D., Tsuribe, P. M. and Landim-Alvarenga, F. C. 2010. Use of a Piezo drill for intracytoplasmic sperm injection into cattle oocytes activated with ionomycin associated with roscovitine. Reprod. Domest. Anim. 45: 654-658. [Medline]

4. Funahashi, H., Cantley, T. C., Stumpf, T. T., Terlouw, S. L. and Day, B. N. 1994. In vitro development of in vitro-matured porcine oocytes following chemical activation or in vitro fertilization. Biol. Reprod. 50: 1072-1077. [Medline] [CrossRef]

5. Haigo, K., Yamauchi, Y., Yazama, F., Yanagimachi, R. and Horiuchi, T. 2004. Full-term development of hamster embryos produced by injection of round spermatids into oocytes. Biol. Reprod. 71: 194-198. [Medline] [CrossRef]

6. Jolliff, W. J. and Prather, R. S. 1997. Parthenogenic development of in vitro-matured, in vivo-cultured porcine oocytes beyond blastocyst. Biol. Reprod. 56: 544-548. [Medline] [CrossRef]

7. Kaufman, M. H. 1983. Methodology: in vitro and in vivo techniques. pp. 20-26. In: Early Mammalian Development: Parthenogenetic Studies (Kaufman M. H. ed.), Cambridge University Press, Cambridge.

8. Kim, N. H., Simerly, C., Funahashi, H., Schatten, G. and Day, B. N. 1996. Microtubule organization in porcine oocytes during fertilization and parthenogenesis. Biol. Reprod. 54: 1397 1404. [Medline] [CrossRef]

9. Kim, N. H., Lee, J. W., Jun, S. H., Lee, H. T. and Chung, K. S. 1998. Fertilization of porcine oocytes following intracytoplasmic spermatozoon or isolated sperm head injection. Mol. Reprod. Dev. 51: 436-444. [Medline] [CrossRef]

10. Kim, N. H., Shin, J. S., Kim, C., Jun, S. H., Lee, H. T. and Chung, K. S. 1999. Fertilization and in vitro development of porcine oocytes following intracytoplasmic injection of round spermatid or round spermatid nuclei. Theriogenology 51: 1441-1449. [Medline] [CrossRef]

11. Kolbe, T. and Holtz, W. 1999. Intracytoplasmic injection
(ICSI) of in vivo or in vitro matured oocytes with fresh ejaculated or frozen-thawed epididymal spermatozoa and additional calcium-ionophore activation in the pig. Theriogenology 52: 671-682. [Medline] [CrossRef]

12. Kolbe, T. and Holtz, W. 2000. Birth of a piglet derived from an oocyte fertilized by intracytoplasmic sperm injection (ICSI). Anim. Reprod. Sci. 64: 97-101. [Medline] [CrossRef]

13. Kubiak, J. Z. 1989. Mouse oocytes gradually develop the capacity for activation during the metaphase II arrest. Dev. Biol. 136: 537-545. [Medline] [CrossRef]

14. Kure-bayashi, S., Miyake, M., Okada, K. and Kato, S. 2000. Successful implantation of in vitro-matured, electro-activated oocytes in the pig. Theriogenology 53: 1105-1119. [Medline] [CrossRef]

15. Kuretake, S., Kimura, Y., Hoshi, K. and Yanagimachi, R. 1996. Fertilization and development of mouse oocytes injected with isolated sperm heads. Biol. Reprod. 55: 789-795. [Medline] [CrossRef]

16. Lacham-Kaplan, O. and Trounson, A. 1995. Intracytoplasmic sperm injection in mice: increased fertilization and development to term after induction of the acrosome reaction. Hum. Reprod. 10: 2642-2649. [Medline]

17. Lai, L., Sun, Q., Wu, G., Murphy, C. N., Kuhholzer, B., Park, K. W., Bonk, A. J., Day, B. B. and Prather, R. S. 2001. Development of porcine embryos and offspring after intracytoplasmic sperm injection with liposome transfected or non-transfected sperm into in vitro matured oocytes. Zygote 9: 339-346. [Medline] [CrossRef]

18. Lee, J. W., Tian, X. C. and Yang, X. 2003. Failure of male pronucleus formation is the major cause of lack of fertilization and embryo development in pig oocytes subjected to intracytoplasmic sperm injection. Biol. Reprod. 68: 1341-1347. [Medline] [CrossRef]

19. Li, G. P., Chen, D. Y., Lian, L., Sun, Q. Y., Wang, M. K., Liu, J. L., Li, J. S. and Han, Z. M. 2001. Viable rabbits derived from reconstructed oocytes by germinal vesicle transfer after intracytoplasmic sperm injection (ICSI). Mol. Reprod. Dev. 58: 180-185. [Medline] [CrossRef]

20. Macháty, Z. and Prather, R. S. 1998. Strategies for activating nuclear transfer oocytes. Reprod. Fertil. Dev. 10: 599-613. [Medline] [CrossRef]

21. Martin, M. J. 2000. Development of in vivo-matured porcine oocytes following intracytoplasmic sperm injection. Biol. Reprod. 63: 109-112. [Medline] [CrossRef]

22. Minamihashi, A., Watson, A. J., Watson, P. H., Church, R. B. and Schultz, G. A. 1993. Bovine parthenogenetic blastocysts following in vitro maturation and oocyte activation with ethanol. Theriogenology 40: 63-76. [Medline] [CrossRef]

23. Nagai, T. 1987. Parthenogenetic activation of cattle follicular oocytes in vitro with ethanol. Gamete Res. 16: 243-249. [Medline] [CrossRef]

24. Nakai, M., Kashiwazaski, N., Takizawa, A., Hayashi, Y., Nakatsukasa, E., Fuchimoto, D., Noguchi, J., Kaneko, H., Shino, M. and Kikuchi, K. 2003. Viable piglets generated from porcine oocytes matured in vitro and fertilized by intracytoplasmic sperm head injection. Biol. Reprod. 68: 1003-1008. [Medline] [CrossRef]

25. Onishi, A., Iwamoto, M., Akita, T., Mikawa, S., Takeda, K., Awata, T., Hanada, H. and Perry, A. C. 2000. Pig cloning by microinjection of fetal fibroblast nuclei. Science 289: 11881190. [Medline] [CrossRef]

26. Ono, T., Mizutani, E., Li, C., Yamagata, K. and Wakayama, T. 2011. Offspring from intracytoplasmic sperm injection of 
aged mouse oocytes treated with caffeine or MG132. Genesis 49: 460-471. [Medline] [CrossRef]

27. Palermo, G., Joris, H., Devroey, P. and Van Steirteghem, A. C. 1992. Pregnancies after intracytoplasmic injection of single spermatozoon into an oocyte. Lancet 340: 17-18. [Medline] [CrossRef]

28. Parrington, J., Swann, K., Shevchenko, V. I., Sesay, A. K. and Lai, F. A. 1996. Calcium oscillations in mammalian eggs triggered by a soluble sperm protein. Nature 379: 364-368. [Medline] [CrossRef]

29. Petters, R. M. and Wells, K. D. 1993. Culture of pig embryos. J. Reprod. Fertil. Suppl. 48: 61-73. [Medline]

30. Prather, R. S., Boice, M. L., Gibson, J., Hoffman, K. E. and Parry, T. W. 1995. In vitro development of embryos from Sinclair miniature pigs: a preliminary report. Theriogenology $\mathbf{4 3}$ : 1001-1007. [Medline] [CrossRef]

31. Prather, R. S., Eichen, P. A., Nicks, D. K. and Peters, M. S. 1991. Artificial activation of porcine oocytes matured in vitro. Mol. Reprod. Dev. 28: 405-409. [Medline] [CrossRef]

32. Prather, R. S., Tao, T. and Machaty, Z. 1999. Development of the techniques for nuclear transfer in pigs. Theriogenology $\mathbf{5 1}$ : 487-498. [Medline] [CrossRef]

33. Prather, R. S., Sims, M. M. and First, N. L. 1989. Nuclear transplantation in early pig embryos. Biol. Reprod. 41: 414418. [Medline] [CrossRef]

34. Procházka, R., Kanka, J., Sutovsky, P., Fulka, J. and Motlik, J. 1992. Development of pronuclei in pig oocytes activated by a single electric pulse. J. Reprod. Fertil. 96: 725-734. [Medline]
[CrossRef]

35. Rho, G. J., Kawarsky, S., Johnson, W. H., Kochhar, K. and Betteridge, K. J. 1998. Sperm and oocyte treatments to improve the formation of male and female pronuclei and subsequent development following intracytoplasmic sperm injection into bovine oocytes. Biol. Reprod. 59: 918-924. [Medline] [CrossRef]

36. Tarkowski, A. K. 1966. An air-drying method for chromosome preparation from mouse eggs. Cytogenesis 5: 394-400. [CrossRef]

37. Tesarik, J. 1996. Fertilization of oocytes by injecting spermatozoa, spermatids and spermatocytes. Rev. Reprod. 1: 149152. [Medline] [CrossRef]

38. Uehara, T. and Yanagimachi, R. 1976. Microsurgical injection of spermatozoa into hamster eggs with subsequent transformation of sperm nuclei into male pronuclei. Biol. Reprod. 15: 467-470. [Medline] [CrossRef]

39. Wallenhorst, S. and Holtz, W. 1999. Transfer of pig embryos to different uterine sites. J. Anim. Sci. 77: 2327-2329. [Medline]

40. Wang, W. H., Abeydeera, L. R., Prather, R. S. and Day, B. N. 1998. Functional analysis of activation of porcine oocytes by spermatozoa, calcium ionophore, and electrical pulse. Mol. Reprod. Dev. 51: 346-353. [Medline] [CrossRef]

41. Zhu, J., Telfer, E. E., Fletcher, J., Springbett, A., Dobrinsky, J. R., De Sousa, P. A. and Wilmut, I. 2002. Improvement of an electrical activation protocol for porcine oocytes. Biol. Reprod. 66: 635-641. [Medline] [CrossRef] 